\title{
Metabolomics of a cell line-derived xenograft model reveals circulating metabolic signatures for malignant mesothelioma
}

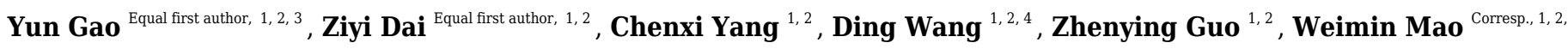 \\ 3 , Zhongjian Chen ${ }^{\text {Corresp. 1, 2, } 3}$ \\ ${ }^{1}$ The Cancer Hospital of the University of Chinese Academy of Sciences (Zhejiang Cancer Hospital), Hangzhou, China \\ 2 Institute of Cancer and Basic Medicine (ICBM), Chinese Academy of Sciences, Hangzhou, China \\ 3 Zhejiang Key Laboratory of Diagnosis\&Treatment Technology on Thoracic Oncology(Lung and Esophagus), Hangzhou, China \\ 4 Zhejiang Chinese Medical University, Hangzhou, China \\ Corresponding Authors: Weimin Mao, Zhongjian Chen \\ Email address: maowm@zjcc.org.cn, chenzj@zjcc.org.cn
}

Background: Malignant mesothelioma (MM) is a rare and highly aggressive cancer. Despite advances in multidisciplinary treatments for cancer, the prognosis for MM remains poor with no effective diagnostic biomarkers currently available. The aim of this study was to identify plasma metabolic biomarkers for better MM diagnosis and prognosis by use of a MM cell line-derived xenograft (CDX) model.

Methods: The MM CDX model was confirmed by hematoxylin and eosin staining and immunohistochemistry. Twenty female nude mice were randomly divided into two groups, 10 for the MM CDX model and 10 controls. Plasma samples were collected two weeks after tumor cell implantation. Gas chromatography-mass spectrometry analysis was conducted. Both univariate and multivariate statistics were used to select potential metabolic biomarkers. Hierarchical clustering analysis, metabolic pathway analysis, and receiver operating characteristic (ROC) analysis were performed. Additionally, bioinformatics analysis was used to investigate differential genes between MM and HC, and survivalassociated genes.

Results: The MM CDX model was successfully established. With VIP $>1.0$ and P-value $<0.05$, a total of 23 differential metabolites were annotated, in which isoleucine, 5-dihydrocortisol, and indole-3-acetamide had the highest diagnostic values based on ROC analysis. These were mainly enriched in pathways for starch and sucrose metabolism, pentose and glucuronate interconversions, galactose metabolism, steroid hormone biosynthesis, as well as phenylalanine, tyrosine and tryptophan biosynthesis. Further, down-regulation was observed for amino acids, especially isoleucine, which is consistent with upregulation of amino acid transporter genes SLC7A5 and SLC1A3 in MM. Overall survival was also negatively associated with SLC1A5, SLC7A5, and SLC1A3.

Conclusion: We found several altered plasma metabolites in MM. The importance of specific metabolic pathways, for example amino acid metabolism, is highlighted herein, although further investigation is warranted. 
1 Title

2 Metabolomics of a cell line-derived xenograft model reveals circulating metabolic

3 signatures for malignant mesothelioma

4 Yun Gao ${ }^{1, \#}$, Ziyi Dai1 ${ }^{1, \#}$, Chenxi Yang ${ }^{1}$, Ding Wang ${ }^{1,2}$, Zhenying Guo $^{1}$, Weimin Mao ${ }^{1, *}$,

5 Zhongjian Chen ${ }^{1, *}$

6 1: The Cancer Research Institute, The Cancer Hospital of the University of Chinese Academy of

7 Sciences (Zhejiang Cancer Hospital), Institute of Cancer and Basic Medicine (ICBM), Chinese

8 Academy of Sciences, Hangzhou, Zhejiang, 310022, China.

9 2: Zhejiang Chinese Medical University, Hangzhou, Zhejiang, 310053, China.

10 \#: These authors contribute equally to this work

$11 *$ Corresponding authors:

12 Zhongjian Chen: The Cancer Research Institute, The Cancer Hospital of the University of

13 Chinese Academy of Sciences (Zhejiang Cancer Hospital), Institute of Cancer and Basic

14 Medicine (ICBM), Chinese Academy of Sciences, Hangzhou, Zhejiang, 310022, China. E-mail:

15 chenzj@zjcc.org.cn.

16 Weimin Mao: The Cancer Research Institute, The Cancer Hospital of the University of Chinese

17 Academy of Sciences (Zhejiang Cancer Hospital), Institute of Cancer and Basic Medicine

18 (ICBM), Chinese Academy of Sciences, Hangzhou, Zhejiang, 310022, China. E-mail:

19 maowm@zjcc.org.cn.

20

21

Abstract

Background: Malignant mesothelioma (MM) is a rare and highly aggressive cancer. Despite advances in multidisciplinary treatments for cancer, the prognosis for MM remains poor with no effective diagnostic biomarkers currently available. The aim of this study was to identify plasma metabolic biomarkers for better MM diagnosis and prognosis by use of a MM cell line-derived xenograft (CDX) model.

Methods: The MM CDX model was confirmed by hematoxylin and eosin staining and immunohistochemistry. Twenty female nude mice were randomly divided into two groups, 10 
29

30

31

for the MM CDX model and 10 controls. Plasma samples were collected two weeks after tumor cell implantation. Gas chromatography-mass spectrometry analysis was conducted. Both univariate and multivariate statistics were used to select potential metabolic biomarkers. Hierarchical clustering analysis, metabolic pathway analysis, and receiver operating characteristic (ROC) analysis were performed. Additionally, bioinformatics analysis was used to investigate differential genes between MM and $\mathrm{HC}$, and survival-associated genes.

Results: The MM CDX model was successfully established. With VIP $>1.0$ and P-value $<0.05$, a total of 23 differential metabolites were annotated, in which isoleucine, 5-dihydrocortisol, and indole-3-acetamide had the highest diagnostic values based on ROC analysis. These were mainly enriched in pathways for starch and sucrose metabolism, pentose and glucuronate interconversions, galactose metabolism, steroid hormone biosynthesis, as well as phenylalanine, tyrosine and tryptophan biosynthesis. Further, down-regulation was observed for amino acids, especially isoleucine, which is consistent with up-regulation of amino acid transporter genes SLC7A5 and SLC1A3 in MM. Overall survival was also negatively associated with SLC1A5, SLC7A5, and SLC1A3.

Conclusion: We found several altered plasma metabolites in MM. The importance of specific metabolic pathways, for example amino acid metabolism, is highlighted herein, although further investigation is warranted.

Keywords: cell line-derived xenograft, GC-MS, malignant mesothelioma, metabolomics, amino acid metabolism

\section{INTRODUCTION}

Malignant mesothelioma (MM) is an uncommon but highly aggressive tumor that is associated with asbestos exposure. The worldwide, age-adjusted mesothelioma mortality rate has increased approximately $5.37 \%$ annually (Delgermaa et al., 2011). It is expected that the ageadjusted mesothelioma incidence and related mortality rate will continue to increase dramatically in the near future, especially in countries where asbestos is still widely used (e.g. China, India, and the Russian Federation) (Carbone et al., 2019). The median survival time for patients with 
56

57

58

59

60

61

62

63

64

65

66

MM is less than 12 months from onset (Ledda, Senia \& Rapisarda, 2018). Treatment is challenging for several reasons, but most important is the difficulty of early stage diagnosis when the patient is typically asymptomatic. In addition, symptoms tend to be vague and often resemble those of other more common diseases such as chest infection (Ahmadzada, Reid \& Kao, 2018). As a result, MM diagnosis is often delayed resulting in inevitable tumor development. Therefore, MM patients urgently need accurate, susceptive, and non-invasive procedures by which to predict, diagnose, and prognosticate disease outcomes.

3

Although cancer is traditionally viewed as a disease of cellular proliferation, more recent studies have proposed cancer as a metabolic disease (Seyfried et al., 2014). Tumors, being highly proliferative, show significant alteration in metabolic pathways, especially in energy production and the biosynthesis of macromolecules (Hammoudi et al., 2011). Therefore, metabolites such as peptides, fatty acids, and steroids in tissues and body fluids (e.g. urine and serum) can provide insight into important disease characteristics.

Metabolomics provides a comprehensive metabolite profile of any biological sample, detecting metabolites involved in the pathophysiology of a disease and serving as a guide for the identification of useful biomarkers (Clish 2015). The most popular methods for metabolomics analysis are gas chromatography-mass (GC-MS), liquid chromatography-mass spectrometry (LC-MS), and nuclear magnetic resonance (NMR) spectroscopy. Metabolomics has the capacity to detect thousands of feature ions at once, which has made it increasingly popular in the cancer research field for biomarker identification (Beger, 2013). In one study of Asian triple negative breast cancer patients, global metabolomics identified altered metabolites that enabled the construction of metabolite-based biomarker panels (Li et al., 2020). As such, metabolomics can be a powerful and useful tool for cancer research. 
83

84

to discover promising metabolite biomarkers to improve early MM diagnosis. Such biomarkers would allow for customized and individualized patient treatment increasing overall survival. In addition, potential survival-related genes were explored by bioinformatics analysis based on the TCGA database. Identified biomarkers and genes may prove to be promising therapeutic targets for the treatment of MM.

\section{MATERIALS AND METHODS}

\section{MM cell line}

Ren cells, a human MM cell line established by (Smythe et al. 1994), were cultured in Gibco $^{\mathrm{TM}}$ Dulbecco's modified eagle medium (DMEM) with L-glutamine (Thermo Fisher Scientific, MA, USA), supplemented with 10\% Fetal Bovine Serum (FBS) (Thermo Fisher Scientific, MA, USA), 100 units $/ \mathrm{ml}$ penicillin, and $100 \mu \mathrm{g} / \mathrm{mL}$ streptomycin (Thermo Fisher Scientific, MA, USA). Cells were incubated at $37^{\circ} \mathrm{C}$ in a $5 \% \mathrm{CO}_{2}$ humidified atmosphere. Trypsin was used for cell collection when confluence reached $80 \%$.

\section{Xenograft model construction}

Animal experimentation was performed under project license (No. 2019-02-010) granted by the Institutional Animal Care and Ethics Committee of Zhejiang Cancer Hospital, in compliance with national or institutional guidelines for the care and use of animals. All animals were housed in individual cages (five mice per cage), with full-value nutritional granulated fodder and distilled water, at $21 \pm 2^{\circ} \mathrm{C}$, at $40 \%-60 \%$ relative humidity, with 12-h light and dark periods.

Twenty female BALB/c nude mice (4-week-old) were purchased from Shanghai SLAC laboratory Animal Co., Ltd. (Shanghai, China) and acclimated for one week before experimentation. The mice were randomly divided into two groups of 10. For the cell-derived xenograft (CDX) model, $200 \mu \mathrm{L}$ of PBS containing $3 \times 10^{6}$ cells were subcutaneously injected into the flanks of mice. The control group received no treatment. Two weeks after transplantation, blood was obtained from the retro-orbital plexus with isoflurane-induced anesthesia. A blood volume of $100 \mu \mathrm{L}$ was collected into a heparinized tube, plasma was separated at 3,000 rpm for 
$11015 \mathrm{~min}$ at $4^{\circ} \mathrm{C}$, and samples were stored at $-80^{\circ} \mathrm{C}$ until analysis. The anesthesia protocol was that 111 provided with the anesthesia machine produced by RWD Life Science Co., Ltd. (GuangDong,

112 China). After experimentation, animal carcasses were loaded into garbage bags and handled by 113 the Institute of Laboratory Animals.

Immunohistochemistry (IHC)

116

117

118

119

120

121

122

123

124

125

126

127

128

Tumors were removed and preserved in 4\% paraformaldehyde. IHC was performed with formalin-fixed paraffin-embedded (FFPE) tissue of tumor samples. Slides were deparaffinized and incubated in 3\% hydrogen peroxide to inactivate endogenous peroxidases. Slides were placed into citric acid repair solution $(\mathrm{pH}=6)$ and boiled at $100^{\circ} \mathrm{C}$ for $90 \mathrm{sec}$ for antigen retrieval. After addition of a protein blocking solution, slides were incubated with antibodies (calretinin, CK5/6, WT1, D2-40, MOC31, SLC1A5, SLC7A5) at $4^{\circ} \mathrm{C}$ overnight, and then incubated with HRP-labeled secondary antibody (Agilent DAKO, CA, USA, catalogue number \#K5007), then the slides were further processed with the DAB regent kit (Agilent DAKO, CA, USA, catalogue number \#K5007). The following antibodies were used; mouse anti calretinin monoclonal antibody (Leica Biosystems, Wetzlar, Germany, catalogue number \#PA0346), mouse anti CK5/6 monoclonal antibody (MXB Biotechnologies, Fujian, China, catalogue number \# MAB-0744), mouse anti WT1 monoclonal antibody (Agilent DAKO, CA, USA, catalogue number \#IR055), mouse anti podoplanin (D2-40) monoclonal antibody (Agilent DAKO, CA, USA, catalogue number \#IR072), mouse anti MOC31 monoclonal antibody (MXB Biotechnologies, Fujian, China, catalogue number \# MAB-0280), rabbit anti SLC1A5 monoclonal antibody (Cell Signaling Technology, MA, USA, catalogue number \#8057), and rabbit anti SLC7A5 polyclonal antibody (Proteintech, CA, USA, catalogue number \# 13752-1AP). Histological features of MM were identified based on the guidelines for the diagnosis and treatment of pleural MM (van Zandwijk et al., 2013). Immunohistochemical analysis was performed on FFPE tissue specimens from MM patients after ethical committee approval (IRB- 
136

137

138

139

140

141

142

143

144

145

146

147

148

149

150

151

152

153

154

155

156

157

158

159

160

161

162

2018-82).

\section{Gas Chromatography/Mass Spectrometry (GC/MS)-based metabolomics profiling}

\section{Sample preparation}

An aliquot of $50 \mu \mathrm{L}$ plasma was mixed with $200 \mu \mathrm{L}$ of methanol (HPLC grade, CNW

Technologies, Duesseldorf, Germany) containing $25 \mu \mathrm{mol} / \mathrm{L}$ 2-chloro-L-phenylalanine

(Shanghai Hengbai Biotech, Shanghai, China) as an internal standard, which was used for normalization. After vortexing for $30 \mathrm{sec}$, samples were ultra-sonicated in iced water for $10 \mathrm{~min}$. After centrifugation at $12000 \mathrm{rpm}$ at $4^{\circ} \mathrm{C}$ for $15 \mathrm{~min}, 180 \mu \mathrm{L}$ of supernatant for each sample were transferred into a new tube and dried in a vacuum concentrator. The residues of each sample were then added with $20 \mu \mathrm{L}$ of $20 \mathrm{mg} / \mathrm{mL}$ methoxyamine hydrochloride (AR grade, TCI, OR, USA) in pyridine (HPLC grade, Adamas Pharmaceuticals, CA, USA). The above samples were kept at $80^{\circ} \mathrm{C}$ for $30 \mathrm{~min}$, after which $40 \mu \mathrm{L}$ of bis-(trimethylsilyl) trifluoroacetamide (BSTFA) in 1\% TMCS (REGIS Technologies, IL, USA) was added and samples were incubated at $70^{\circ} \mathrm{C}$ for $1.5 \mathrm{~h}$. The samples were stored at room temperature until analysis. Quality control (QC) samples were prepared by pooling an aliquot of $30 \mu \mathrm{L}$ from each sample.

\section{GC-TOF-MS Analysis}

Metabolomics was performed on an Agilent 7890 gas chromatograph system equipped with Pegasus HT time-of-flight mass spectrometer (LECO, St. Joseph, MI, USA). Chromatographic separation was achieved on a DB-5MS capillary column $(30 \mathrm{~m} \times 0.25 \mathrm{~mm}, 0.25 \mu \mathrm{m}$ film thickness, J\&W Scientific, Folsom, CA, USA). The GC-TOF-MS settings were as follows: $1 \mu \mathrm{L}$ sample was loaded in a splitless mode, rate $3 \mathrm{~mL} / \mathrm{min}$ for the front inlet purge flow rate, 1 $\mathrm{mL} / \mathrm{min}$ for the gas flow rate; the initial temperature was $50^{\circ} \mathrm{C}$, then increased to $310^{\circ} \mathrm{C}$ with a rate of $20^{\circ} \mathrm{C} / \mathrm{min}$, then kept at $310^{\circ} \mathrm{C}$ for $6 \mathrm{~min}$; the temperatures for injection, transfer line, and ion source were 280,280 , and $250^{\circ} \mathrm{C}$, respectively; the electron impact energy was set as $70 \mathrm{eV}$; full-scan mode with a mass-to-charge ratio range from 50 to 500 was used in mass collection. 
164

165

166

167

168

169

170

171

172

173

174

175

176

177

\section{Metabolomics data analysis}

GC-TOF-MS raw data were processed by Chroma TOF 4.3X software (LECO Corporation, St. Joseph, MI, United States). The LECO-Fiehn Rtx5 database was used for data extraction and analysis. Normalization was performed for each sample through calculating the ratios of peaks of analytes and 2-chloro-L-phenylalanine, and relative quantification was applied in further comparison between xenograft and control groups. Metabolite identification was achieved by matching both mass spectra and retention index of the detected metabolic features. Peaks that were not detected in more than half of QC samples or peaks with RSD $>30 \%$ in QC samples were removed, a dataset with 20 samples and identified metabolites was obtained.

Both univariate and multivariate analysis were conducted to select the differential metabolites. $\log _{2}$ (Fold changes) $\left(\log _{2}(\mathrm{FC})\right)$ of each metabolite between xenograft and control groups were calculated through dividing their average values. $\log _{10}$ (P-value) was calculated for each metabolite using a two-tailed Student's t-test. Besides, component analysis (PCA) of all the identified metabolites was used to observe the clusters of samples. Partial least squaresdiscriminant analysis (PLS-DA) was performed to select the most significant differential metabolites between xenograft and controls using R package "ropls" (version 1.18.8), and variable importance in projection (VIP) of each metabolite was obtained from PLS-DA. Permutation test with cross validation for 20 times was applied to test reliability of the PLS-DA model. Volcano plot with $\log 2(\mathrm{FC})$ and $-\log 10$ (P-value) values was used to show the differential metabolites between xenograft and control groups.

\section{Hierarchical clustering analysis and metabolic pathway analysis}

Hierarchical clustering analysis was performed based on relative abundance of differential metabolites between MM and control groups. Results were illustrated as a heatmap using R package "pheatmap". Pathway analysis of the differential metabolites was performed with an online tool (https://www.metaboanalyst.ca/). Top 25 pathways with P-value $<0.05$ were selected 
190

191

192

193

194

195

based on enrichment ratio (i.e., observed hits/expected hits).

\section{Correlation analysis and receiver operating characteristics (ROC) analysis}

Pearson correlation analysis was performed for each pair of differential metabolites, the results were visualized using R package "corrplot".

ROC analyses were performed for all selected differential metabolites using all samples. Based on area under curve (AUC), top 6 metabolites were selected and their relative levels in $\mathrm{MM}$ and control were shown as box plots. R package " $p R O C$ " was used for ROC analyses and package "ggplot2" was used for visualization.

\section{Bioinformatics analysis for amino acid transporter genes}

Gene array dataset (GSE51024) from 55 MM tumor samples and along with paired normal tissue (for 41 tumors) was obtained from GEO database (https:/www.ncbi.nlm.nih.gov/geo). Statistical analysis was performed using $\mathrm{R}$ package "limma" to screen for statistically differentially expressed genes $(\mathrm{P}-\mathrm{value}<0.05)$ and to calculate fold changes of selected genes. mRNA expression in type of RNA-seq count and corresponding survival data for $86 \mathrm{MM}$ patients from Cancer Genome Atlas (TCGA) were downloaded from GDC TCGA mesothelioma dataset in UCSC Xena TCGA hub (https://xenabrowser.net/). Amino acid transporter gene list was based on Bröer (2020). Samples were divided into high and low groups according to median value of each gene as cut-off value. Kaplan-Meier plots with log-Rank test were performed to determine significant differences between the two groups. Finally, univariate Cox proportional hazards regression analysis was performed to determine relationships among amino acid transporter genes and prognosis. P-value $<0.05$ was considered significant.

\section{RESULTS}

\section{CDX model information}


biphasic MM, characterized by both epithelioid cell structure and sarcomatous components

218

219

220

221

222

223

\section{4}

225

226

227

228

229

230

231

232

233

234

235

236

237

238

239

240

241

242

(Figure 1A). IHC characteristics were consistent with MM clinico-pathological features, including diffuse positivity for calretinin (Figure 1B) and focal positivity for WT1 (Figure 1C) for mesothelioma, focal positivity for CK5/6 (Figure 1D), wide positivity for D2-40 (Figure 1E) for epithelial carcinoma, and negative for MOC31 (Figure 1F) adenocarcinoma. The results demonstrate the MM cell line-derived xenograft model to be successfully established.

\section{Metabolic differences between the MMxenograft group and the control group}

A total of 377 peaks were obtained, and 187 metabolites were annotated for further multivariate analysis. The score plots of the first two principal components (PC1/PC2) in PCA showed an overlap between the xenograft model and control group, while PLS-DA analysis revealed a clear separation between the xenograft model and control, with a $R^{2} Y$ value of 0.910 and a $\mathrm{Q}^{2}$ value of 0.371 (Figure 2A, B). Permutation tests further revealed the current PLS-DA model to be reliable (Figure 2C). After application of filtering criteria, a total of six up-regulated metabolic features and 42 down-regulated metabolic features were obtained (Figure 2D).

\section{Differential metabolic patterns and pathway enrichment}

After annotation, 23 metabolites were obtained, including 6 up-regulated and 17 downregulated (Table 1). Based on these differential metabolites, the CDX group showed a distinctive metabolic pattern that differed from the control group (Figure 3A). Based on all differential metabolites, 19 metabolic pathways were enriched. Steroid hormone biosynthesis was the most significantly enriched pathway with three hits, followed by starch and sucrose metabolism, pentose and glucuronate interconversions, as well as phenylalanine, tyrosine, and tryptophan biosynthesis pathways (Table 2).

\section{Diagnostic value of plasma metabolites}


Figure 4A shows the Spearman correlations for the differential metabolites based on their

244

245

246

247

248

249

250

251

252

253

254

255

256

257

258

259

260

261

262

263

264

265

266

267

268

269

relative circulating levels. ROC analysis identified the top six metabolites with the highest $\mathrm{AUC}_{\mathrm{ROC}}$ values. They were isoleucine (AUC: 0.860), 5-dihydrocortisol (AUC: 0.860), guanosine (AUC: 0.850), indole-3-acetamide (AUC: 0.850), trehalose-6-phosphate (AUC: 0.840), and diglycerol (AUC: 0.830) (Figure 4B-G). Of these, indole-3-acetamide and diglycerol were upregulated in the MM xenograft model, with the other four down-regulated.

\section{Dysregulated amino acid metabolism}

In the plasma of CDX model mice, most of the detected amino acids were downregulated including isoleucine, valine, and proline, with the exception of tyrosine (Figure 5A). Isoleucine, which was decreased in the mesothelioma model, had diagnostic value (Figure 4B).

The bioinformatics analysis identified many prognosis-related genes. These genes were compared to a list of amino acid transporter genes in the referenced paper, with overlap mainly with SLC transporter genes. Survival analysis of the SLC genes indicated that the three most significant genes were $S L C 1 A 5(\mathrm{HR}=1.70), S L C 7 A 5(\mathrm{HR}=2.35)$, and $S L C 1 A 3(\mathrm{HR}=2.21)$ (Figure 5B). All of the most significant SLC genes had hazard ratios over one, indicating them to be unfavorable prognostic factors (Table 3). Data from GEO (Table S1) showed that gene expressions of amino acid transporters $S L C 7 A 5$ and $S L C 1 A 3$ were significantly upregulated, whereas $S L C 1 A 5$ was downregulated in tissues of MM patients compared to controls. Immunohistochemistry also validated expressions of SLC1A5 and SLC7A5 in MM tumor specimens from both mouse xenografts (Figure S1A, C) and MM patients (Figure S1B, D).

\section{DISCUSSION}

By using a MM cell line, we constructed a xenograft model with pathological characteristics highly consistent with that of MM. Strong positive expression of calretinin and negative expression of MOC31 are compatible with the pathological features of MM cases (van Zandwijk $N$, 2013). Thus, the Ren cell line xenograft model is a reliable tool, readily available for research 
270

271

272

273

274

275

276

277

278

279

280

281

282

283

284

285

286

287

288

289

290

291

292

293

294

295

296

purposes.

Oncogenic mutations in tumor cells can induce a metabolic imbalance in native homoeostasis. Our study, based on GC-MS metabolomics, identified 23 differentially expressed plasma metabolites for the CDX group and the control group. The PLS-DA model showed good separation for the model group and the healthy control group. The most notable metabolites are discussed below.

It is widely acknowledged that some cancers are hormone-dependent (e.g., breast and prostate cancer) (Key, 1995), whereas no study reported hormones as carcinogens for MM. Nevertheless, we detected changes in plasma levels of some hormones. We observed 5dyhydrocortisol and hydrocortisone levels to be significantly decreased. Hydrocortisone is a steroid hormone that is typically anti-inflammatory and is used for treatment of cancers, such as leukemia. Asbestos can induce inflammatory changes, including increase in MMP7, CXCR5, CXCL13, and CD44, and this chronic inflammation can lead to chronic diseases, such as cancer (Kumagai-Takei et al. 2018). During chronic inflammation, pro-inflammatory metabolites are upregulated to create an ideal environment for tumour growth (Rayburn, Ezell \& Zhang, 2009). Therefore, we speculate that hydrocortisone exhibits an anti-tumour effect by reducing the inflammatory response within the tumor (Linton et al., 2012).

It should be noted that cholesterol, which can be used to produce hydrocortisone, was also decreased. This supports our speculation that hydrocortisone was depleted in diseased tissue. Meta-analysis studies conducted on patients with low cholesterol have shown an association with low cholesterol levels and a high risk for cancer. However, a causal relationship has not been established. Low cholesterol may not be a risk factor for cancer, rather it may be a sign of undiagnosed cancer (Tanne, 2007). Therefore, we speculate that cancer can lower cholesterol levels. A decrease in cholesterol levels may be a result of increased uptake by tumour cells. 
297 Cholesterol is a key component of cell membranes and is essential to cell

298 proliferation(Fernández, de Cedrón \& de Molina, 2020). Another significantly altered lipid was

299 glycerol. Glycerol is component of triacylglycerol. In colorectal cancer, total lipid content is

300

301

302

303

304

305

306

307

308

309

310

311

312

313

314

315

316

317

318

319

320

321

322

323

reduced (Mika et al., 2020). Herein, we speculate that tumor cells dysregulate lipid metabolism, leading to increased oxidation of triacylglycerol in order to produce more energy for tumor growth, consequently releasing more glycerol into the circulation.

Isoleucine is also decreased significantly. Isoleucine is an essential amino acid (EAA) for mammals, used for biosynthesis, energy production, and as a mediator of redox balance (Lieu et al., 2020). Amino acids can provide both nitrogen and carbon for necessary biosynthesis of molecules such as purines as well as serve as an alternative to glucose for the energy necessary in tumor development and cancer cell proliferation. Amino acids are also involved in activities such as NADPH production and glutathione synthesis, essential to redox hemostasis in cancer cells (Vučetić et al., 2017). Isoleucine was also reported to upregulate in pancreatic ductal adenocarcinoma (PDAC) (Jiang et al., 2021), so we speculate that the decrease in isoleucine in our study is due to constant cancer cell uptake. The high demand for EAAs is also reflected in the upregulation of EAA transporters that are evident in many cancers (Lieu et al., 2020). In MM, survival analysis of amino acid transporter genes SLC1A5, SLC7A5, and SLC1A3 were found to be unfavorable prognostic factors, consistent with previous findings. In addition, expressions of SLC7A5 and SLC1A3 were significantly upregulated in MM based on bioinformatic analysis, indicating its prominence to be a therapeutic target. On contrary, SLC1A5 was downregulated, which is inconsistent with results from other studies, therefore more research is needed to validate this trend. Isoleucine is an important branched chain amino acid (BCAAs). Such amino acids are essential for protein synthesis, as a source of nitrogen, and for energy production, all of which are vital for tumor growth (Ananieva \& Wilkinson, 2018). BCAAs can also donate nitrogen for glutamate synthesis, which can then be used to synthesize glutamine, important to tumor growth and maintenance (Hutson, Sweatt \& LaNoue, 2005). The process is catalyzed by 
324 branched-chain aminotransferase 1 (BCAT1) and mitochondrial branched-chain

325 aminotransferase 2 (BCAT2). Recent evidence has shown that these enzymes are overexpressed

326 in many cancers (Ananieva \& Wilkinson, 2018). Survival analysis of these enzymes by GEPIA at

327 a quartile cut-off level demonstrated a significant difference between the high and low

328 expression groups. High expression was associated with a poor prognosis, consistent with our 329 findings.

330

331

332

333

334

335

336

337

338

339

340

341

342

343

344

345

346

347

348

349

350

In this study, we found that citrate levels were significantly decreased in the cancer model group compared to the control group. Recent studies have suggested elevated consumption of citrate is a consequence of higher metabolic demand in cancer cells (Haferkamp et al., 2020). Accordingly, we assume that cellular uptake of citrate increased during tumor growth.

\section{CONCLUSIONS}

Metabolic changes in cancer have long been acknowledged and considered as candidate therapeutic targets. The proposed mechanisms above remain hypothetical and need further investigation to validate, but the findings may provide for better diagnosis and prognostication for MM. We believe these results provide insight into mesothelioma metabolic reprogramming and may provide targets that can be exploited for therapeutic use.

\section{Author contribution:}

Study concept and design: Z.C. and W.M.; CDX model construction: Y.G. and Z.C.; CDX model confirmation: Z.G.; acquisition, analysis, or interpretation of data: Y.G., Z.D., and C.Y.; writing and drafting of the manuscript: Y.G., C.Y., and Z.D.; critical revision of the manuscript for important intellectual content, Z.C., W.M., D.W., C.Y., and Z.D. All authors have read and agreed to the published version of the manuscript.

\section{Data Availability}


351

352

\section{3}

354

355

356

\section{7}

358

359

360

361

362

363

364

365

366

367

368

369

370

371

372

373

374

375

376

377

378

379

380

381

382

383

384

385

386

387

The raw measurements are available in the Supplemental file.

\section{Acknowledgement:}

We thank International Science Editing (http://www.internationalscienceediting.com) for editing this manuscript.

\section{Conflicts of Interest:}

The authors declare no conflict of interest.

\section{References}

Ahmadzada T, Reid G, Kao S. 2018. Biomarkers in malignant pleural mesothelioma: current status and future directions. Journal of Thoracic Disease 10(Suppl 9):S1003.

Ananieva EA, Wilkinson AC. 2018. Branched-chain amino acid metabolism in cancer. Current Opinion in Clinical Nutrition and Metabolic Care 21(1):64-70.

Beger RD. 2013. A review of applications of metabolomics in cancer. Metabolites 3(3):552-574. Carbone M, Adusumilli PS, Alexander HR Jr, Baas P, Bardelli F, Bononi A, Bueno R, FelleyBosco E, Galateau-Salle F, Jablons D, Mansfield AS, Minaai M, de Perrot M, Pesavento P, Rusch V, Severson DT, Taioli E, Tsao A, Woodard G, Yang H, Zauderer MG, Pass HI. 2019. Mesothelioma: Scientific clues for prevention, diagnosis, and therapy. $C A: A$ Cancer Journal for Clinicians 69(5):402-429.

Clish CB. 2015. Metabolomics: an emerging but powerful tool for precision medicine. Molecular Case Studies 1(1):a000588.

Delgermaa V, Takahashi K, Park EK, Le GV, Hara T, Sorahan T. 2011. Global mesothelioma deaths reported to the World Health Organization between 1994 and 2008. Bulletin of the World Health Organization 89:716-724.

Fernández LP, de Cedrón MG, de Molina AR. 2020. Alterations of lipid metabolism in cancer: implications in prognosis and treatment. Frontiers in Oncology 10:577420.

Haferkamp S, Drexler K, Federlin M, Schlitt HJ, Berneburg M, Adamski J, Gaumann A, Geissler EK, Ganapathy V, Parkinson EK, Mycielska ME. 2020. Extracellular citrate fuels cancer cell metabolism and growth. Frontiers in Cell and Developmental Biology 8:1518.

Hammoudi N, Ahmed KB, Garcia-Prieto C, Huang P. 2011. Metabolic alterations in cancer cells and therapeutic implications. Chinese Journal of Cancer 30(8):508.

Hutson SM., Sweatt AJ, LaNoue KF. 2005. Branched-chain amino acid metabolism: implications for establishing safe intakes. The Journal of Nutrition 135(6):1557S-1564S.

Key TJ. 1995. Hormones and cancer in humans. Mutation Research/Fundamental and Molecular Mechanisms of Mutagenesis 333(1-2):59-67.

Ledda C, Senia, P, Rapisarda V. 2018. Biomarkers for early diagnosis and prognosis of 
388

389

390

391

392

393

394

395

396

397

398

399

400

401

402

403

404

405

406

407

408

409

410

411

412

413

414

415

416

417

418

malignant pleural mesothelioma: The quest goes on. Cancers 10(6):203.

Li L, Zheng X, Zhou Q, Villanueva N, Nian W, Liu X, Huan T. 2020. Metabolomics-based discovery of molecular signatures for triple negative Breast cancer in Asian female population. Scientific Reports 10(1):1-12.

Lieu EL, Nguyen T, Rhyne S, Kim J. 2020. Amino acids in cancer. Experimental \& Molecular Medicine 52(1):15-30.

Kumagai-Takei N, Yamamoto S, Lee S, Maeda M, Masuzzaki H, Sada N, Yu M, Yoshitome K, Nishimura Y, Otsuki T. 2018. Inflammatory Alteration of Human T Cells Exposed Continuously to Asbestos. Int J Mol Sci. 19(2): 504.

Linton A, van Zandwijk N, Reid G, Clarke S, Cao C, Kao S. 2012. Inflammation in malignant mesothelioma-friend or foe? Annals of Cardiothoracic Surgery 1(4):516-522.

Mika A, Pakiet A, Czumaj A, Kaczynski Z, Liakh I, Kobiela J, Perdyan A, Adrych K, Makarewicz W, Sledzinski T. 2020. Decreased triacylglycerol content and elevated contents of cell membrane lipids in colorectal cancer tissue: a lipidomic study. Journal of Clinical Medicine 9(4):1095.

Rayburn ER, Ezell SJ, Zhang R. 2009. Anti-inflammatory agents for cancer therapy. Molecular and Cellular Pharmacology 1(1):29-43.

Seyfried TN, Flores RE, Poff AM, D’Agostino DP. 2014. Cancer as a metabolic disease: implications for novel therapeutics. Carcinogenesis 35(3):515-527.

Tanne JH. 2007. Meta-analysis says low LDL cholesterol may be associated with greater risk of cancer. British Medical Journal Publishing Group.

van Zandwijk N, Clarke C, Henderson D, Musk AW, Fong K, Nowak A, Loneragan R, McCaughan B, Boyer M, Feigen M, Currow D, Schofield P, Nick Pavlakis BI, McLean J, Marshall H, Leong S, Keena V, Penman A. 2013. Guidelines for the diagnosis and treatment of malignant pleural mesothelioma. Journal of Thoracic Disease 5(6):E254E307.

Vučetić M, Cormerais Y, Parks SK, Pouysségur J. 2017. The central role of amino acids in cancer redox homeostasis: vulnerability points of the cancer redox code. Frontiers in Oncology 7:319.

Jiang W, Qiao L, Han Y, Zhang A, An H, Xiao J, Ren L. 2021. Pancreatic stellate cells regulate branched-chain amino acid metabolism in pancreatic cancer. Ann Transl Med. 9(5): 417. 


\section{Figure 1}

Histopathology (HE) and immunohistochemistry (IHC) findings in tumors from malignant mesothelioma xenograft mice.

(A) Representative images of H\&E staining, the tumor cells were morphologically diverse, large in size, and markedly heteromorphic. (B-C) IHC staining with mesothelioma markers Calretinin and WT1 were diffuse positive and focal positive. (D-E) IHC staining with epithelial carcinoma markers CK5/6 and D2-40 were focal positive and widely positive. (F) IHC staining of adenocarcinoma marker MOC31 was negative. (magnification: HE, $\times 400$; IHC, $\times 400$ ). 

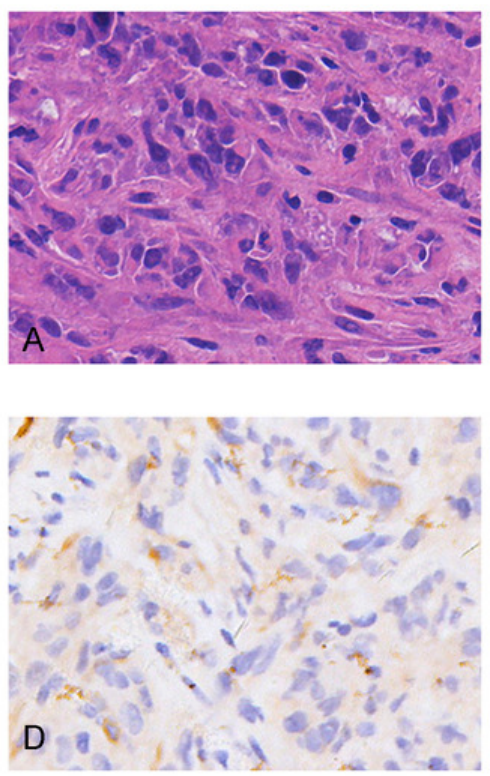
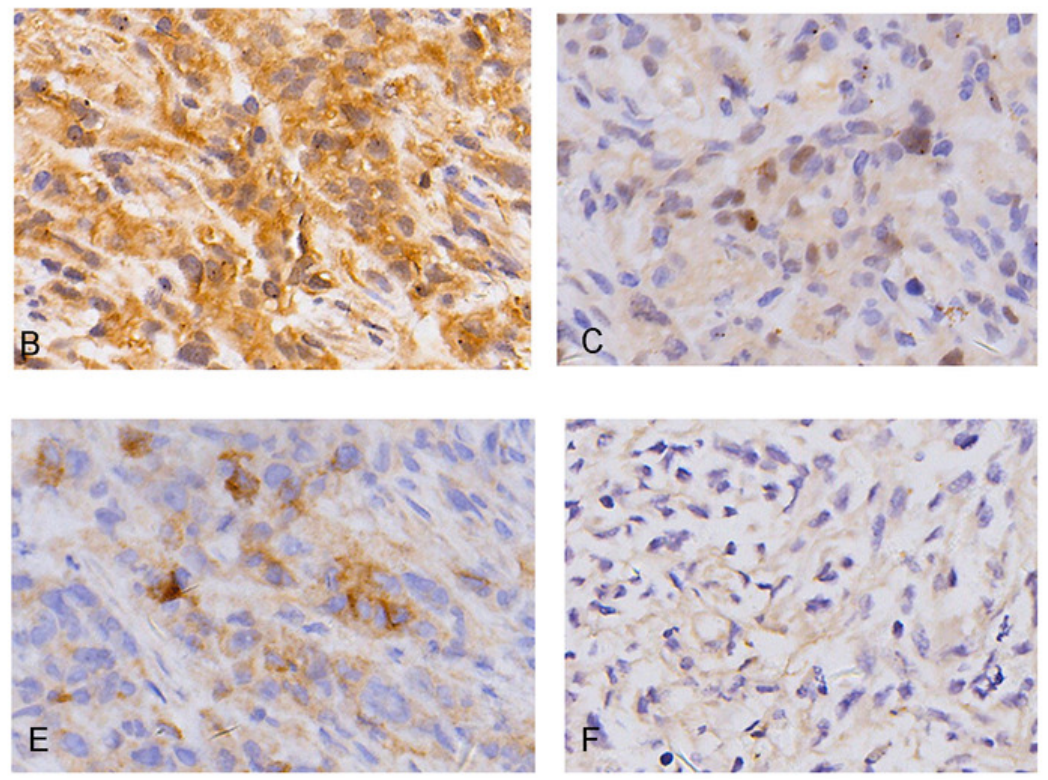


\section{Figure 2}

Multivariate statistical analysis of metabolites.

(A) Score scatter plot of PCA analysis for plasma metabolomics between xenograft mice and controls. T represent the xenograft mice group, and $\mathrm{N}$ is the control group. (B) Score scatter plot of PLS-DA model for group xenograft mice and controls. The abscissa PC1 represents the first principal component, and the ordinate PC2 represents the second principal component. (C) Permutation test of PLS-DA modeling. (D) Volcano plots showing differential feature ions in model mice vs. controls. Differential ions were defined by VIP $>1.0$ and P-value $<0.05$; ions with Log2(Fold Change) $>0$ were considered as upregulated, vice versa. Significantly up-regulated ions are indicated in red, significantly down-regulated ions are indicated in blue, and non-significant differences in metabolites are grey. 
A

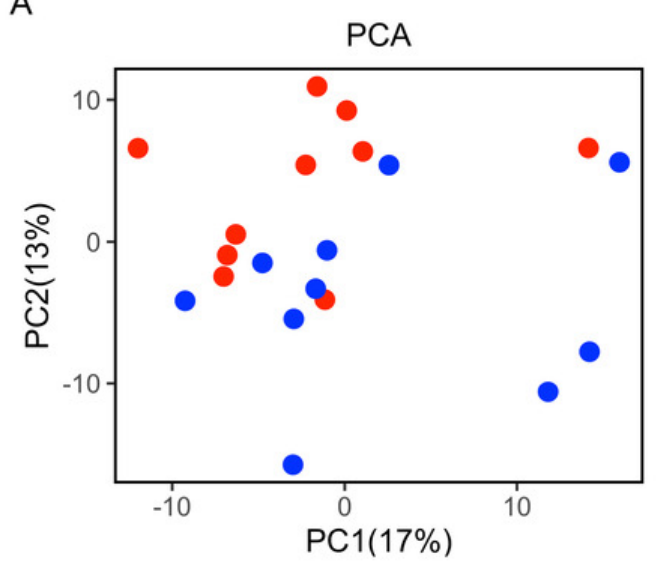

C

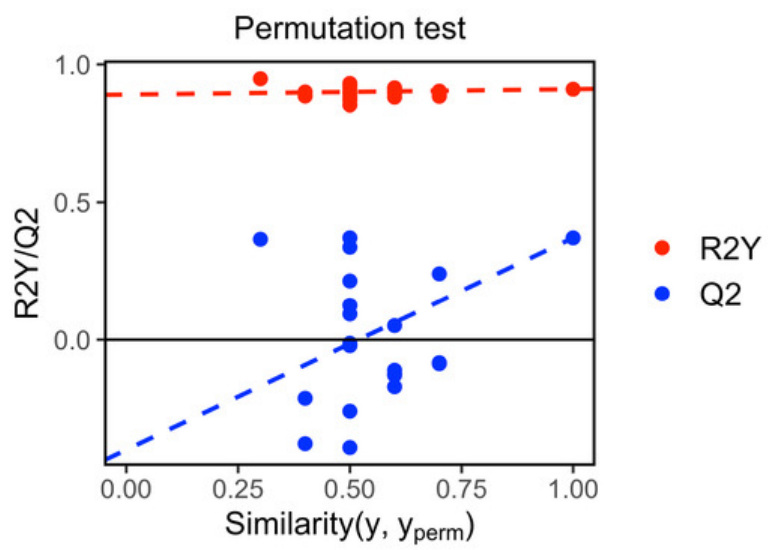

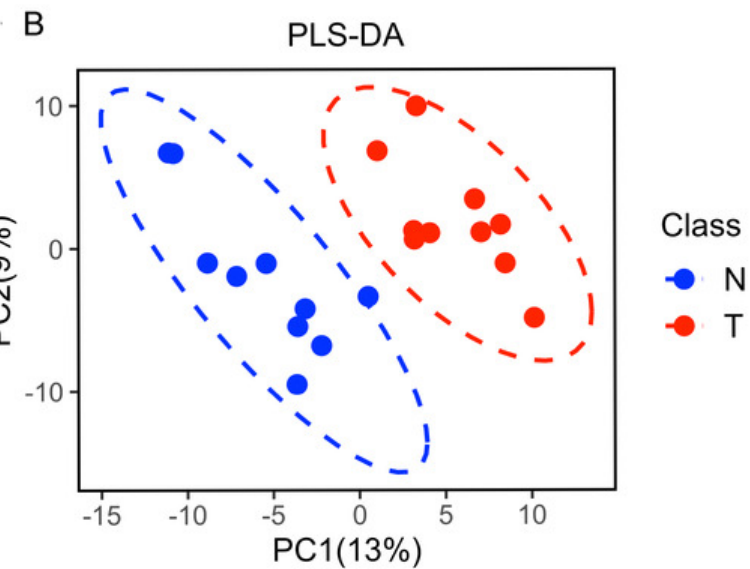

D

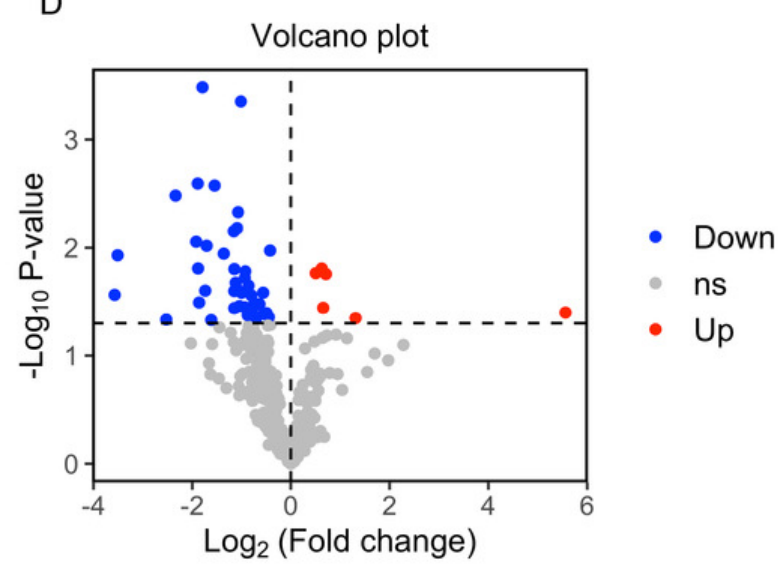




\section{Figure 3}

Heatmap from hierarchical clustering analysis and a diagram of the metabolic pathway enrichment analysis.

(A) Heatmap comparing levels of metabolites of model mice group and the control group. A single mouse corresponded to each column of the heatmap. The mice of model group are labeled by red color, and the controls are green. The color scale indicates the relative expression levels of the metabolites across all samples, blue represents an expression less than the mean, while red represents a higher expression level greater than the mean. (B) Pathway analysis of the metabolites in model mice group. 
A

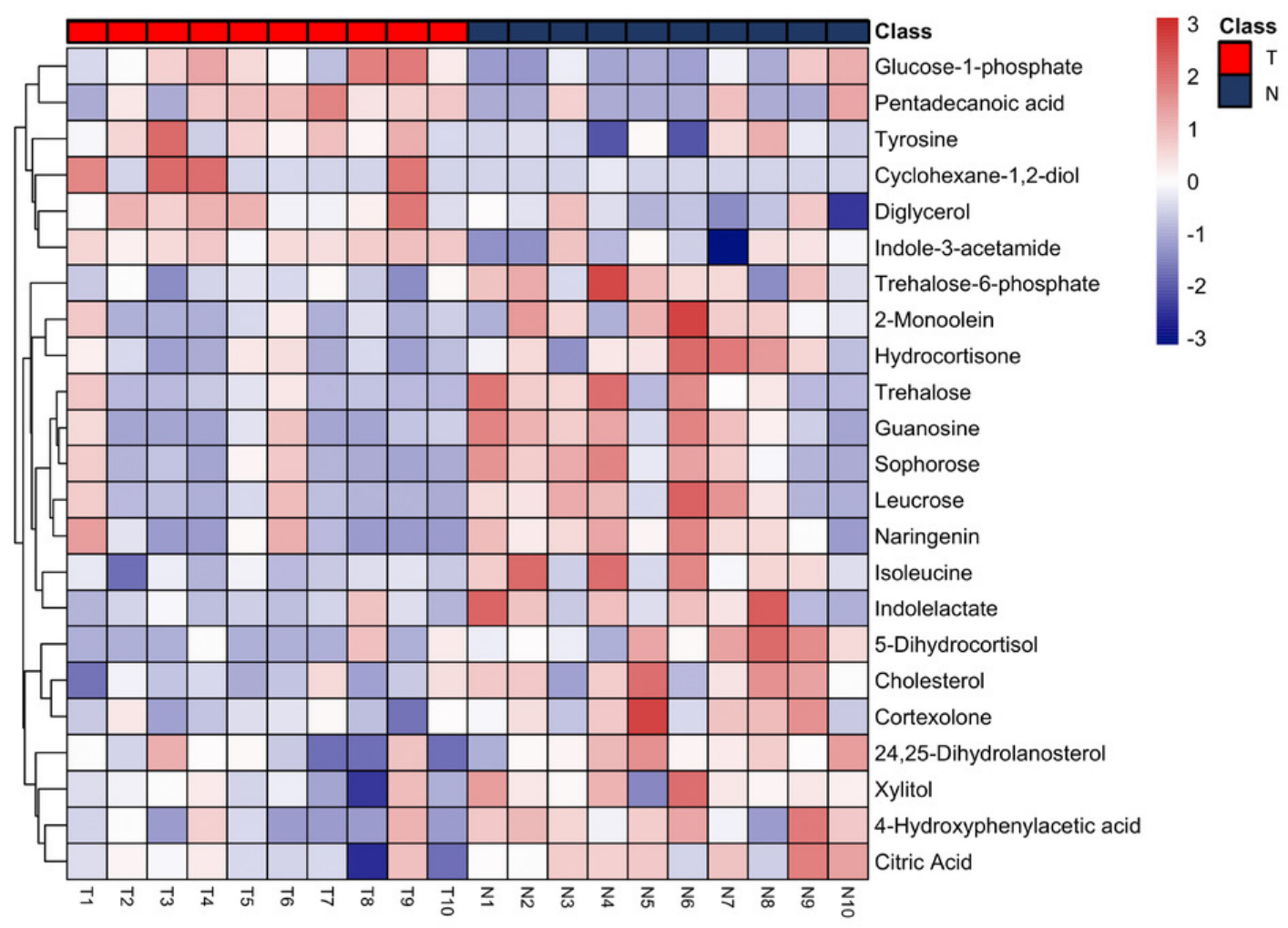

B

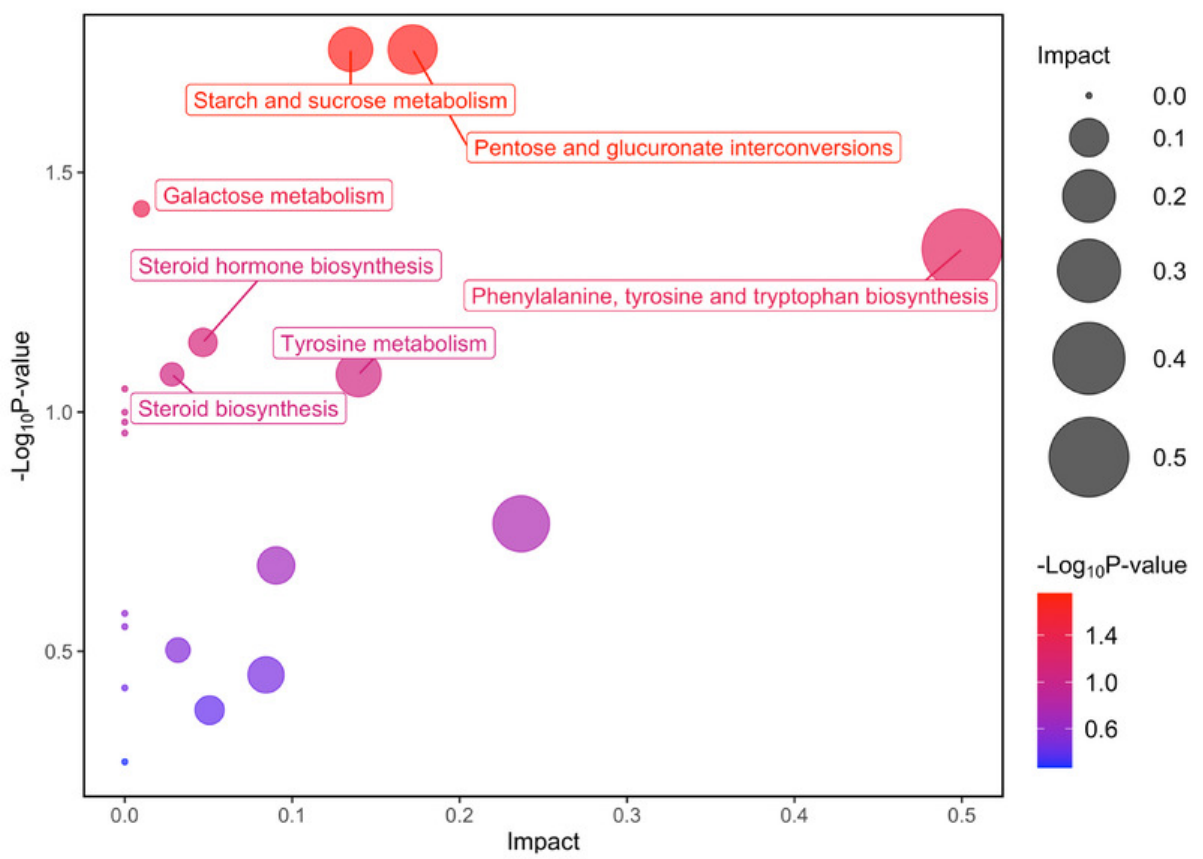




\section{Figure 4}

Metabolite-based correlation analyses and ROC analyses.

(A) Correlation pattern among measured metabolites over the entire cohort using pearson correlation. (B-G) Receiver operating characteristic (ROC) curves of the six differential metabolites with highest area under curve (AUC) values: Isoleucine, lyxonic acid, 5dihydrocortisol, guanosine, indole-3-acetamide, and trehalose-6-phosphate; and boxplots comparing relative intensities of these metabolites between cell-derived xenograft (CDX) and control groups. FC: fold change; P-value was calculated from a two-tailed Student's t-test, and P-value $<0.05$ was considered as significant. 

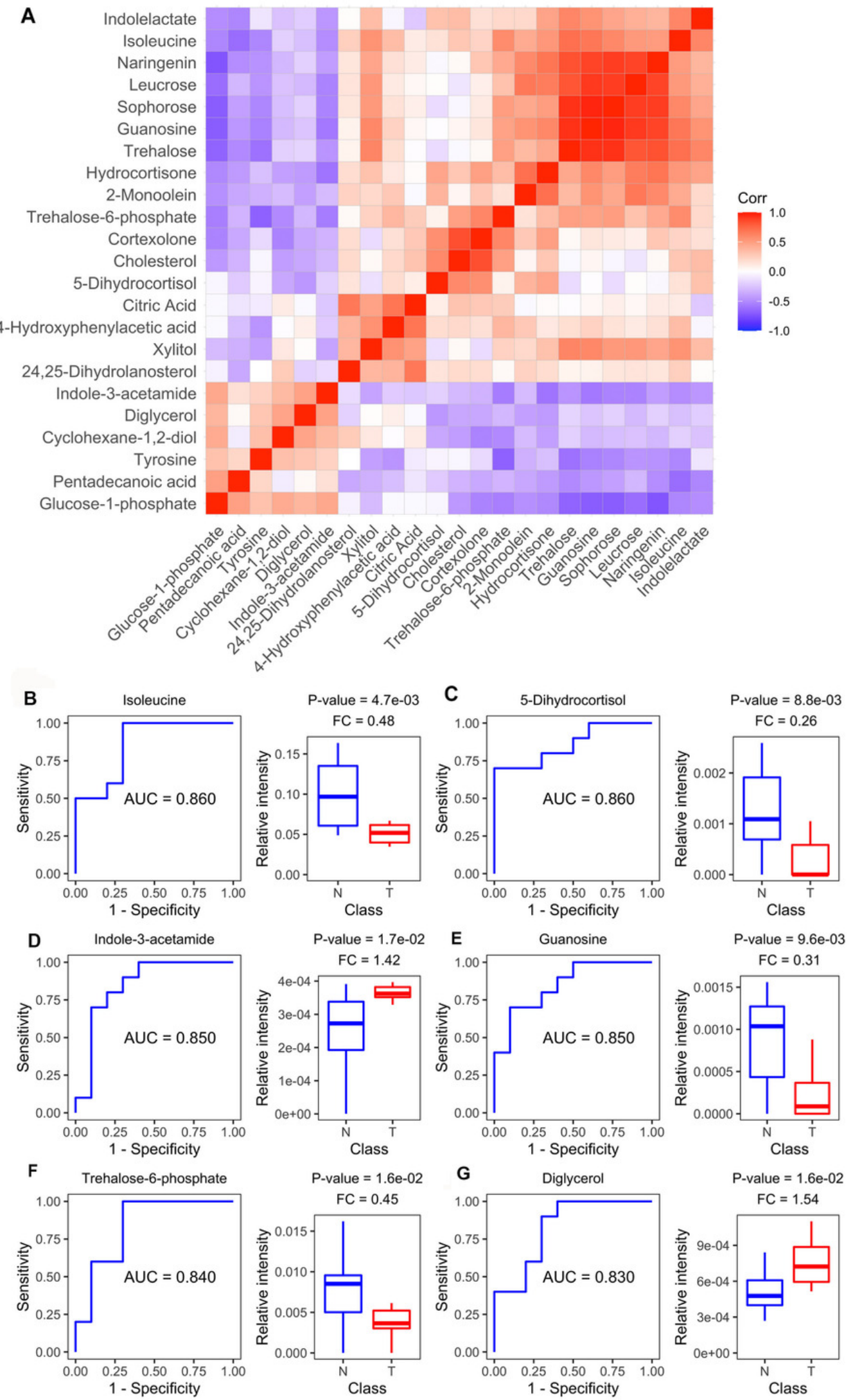


\section{Figure 5}

Differential amino acids in MM and survival-related transporters.

(A) Heatmap comparing the amino acids of samples in the model mice group and the controls. The color scale indicates the relative expression levels of the amino acids, blue represents an expression level less than the mean, while red represents an expression level greater than the mean. (B-D) Kaplan-Meier survival curves for patients with mesothelioma grouped by the different expression levels of SLC1A5 (B), SLC7A5 (C), SLC1A3 (D). 


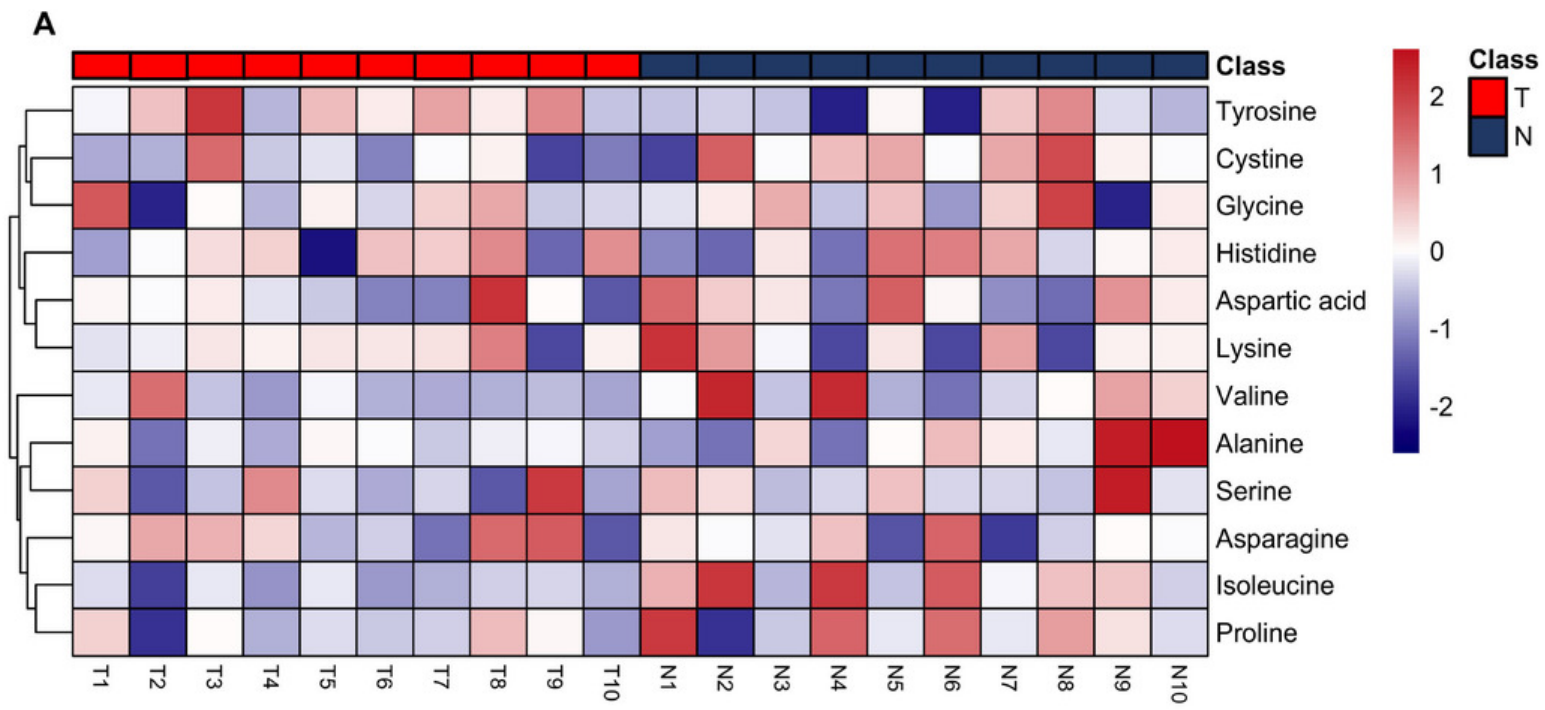

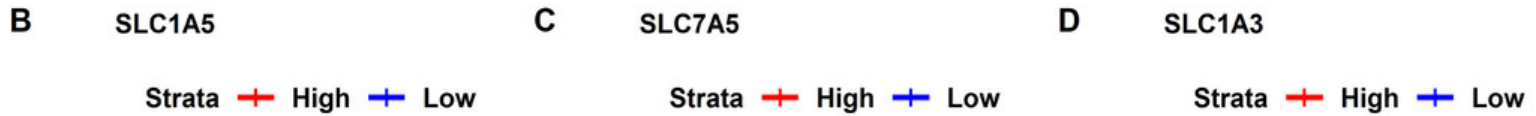
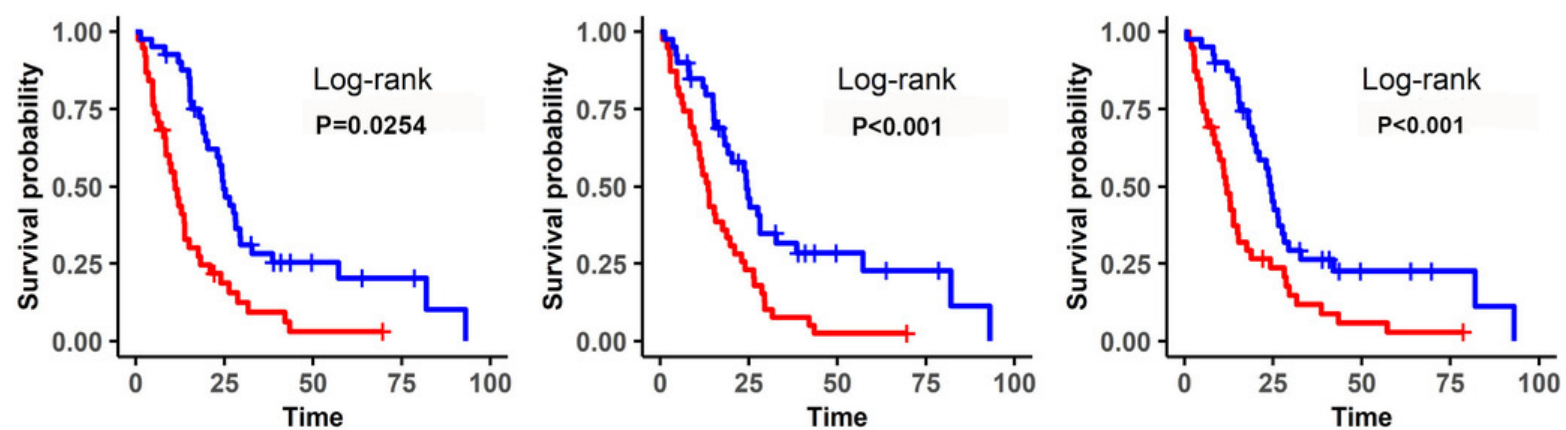


\section{Table 1 (on next page)}

List of identified differential metabolites between model group and healthy controls. 
1

\begin{tabular}{cccccc}
\hline No & Metabolite & VIPa & P-value & Fold change $^{\mathbf{b}}$ & AUC $_{\text {ROC }}{ }^{\mathbf{c}}$ \\
\hline 1 & Isoleucine & 1.9 & $4.70 \mathrm{E}-03$ & 0.48 & 0.860 \\
2 & 5-Dihydrocortisol & 2.1 & $8.10 \mathrm{E}-03$ & 0.26 & 0.860 \\
3 & Guanosine & 1.8 & $8.80 \mathrm{E}-03$ & 0.31 & 0.850 \\
4 & Cholesterol & 1.8 & $9.60 \mathrm{E}-03$ & 0.75 & 0.800 \\
5 & 4-Hydroxyphenylacetic acid & 1.8 & $1.13 \mathrm{E}-02$ & 0.39 & 0.810 \\
6 & Cortexolone & 1.7 & $1.42 \mathrm{E}-02$ & 0.53 & 0.800 \\
7 & Diglycerol & 1.8 & $1.49 \mathrm{E}-02$ & 1.54 & 0.830 \\
8 & Trehalose-6-phosphate & 1.7 & $1.57 \mathrm{E}-02$ & 0.45 & 0.840 \\
9 & Indole-3-acetamide & 1.7 & $1.72 \mathrm{E}-02$ & 1.42 & 0.850 \\
10 & Glucose-1-phosphate & 1.6 & $1.75 \mathrm{E}-02$ & 1.64 & 0.820 \\
11 & Sophorose & 1.6 & $2.04 \mathrm{E}-02$ & 0.46 & 0.800 \\
12 & 2-Monoolein & 1.7 & $2.16 \mathrm{E}-02$ & 0.3 & 0.820 \\
13 & Hydrocortisone & 1.6 & $2.23 \mathrm{E}-02$ & 0.45 & 0.780 \\
14 & Leucrose & 1.6 & $2.24 \mathrm{E}-02$ & 0.46 & 0.770 \\
15 & Citric acid & 1.6 & $2.54 \mathrm{E}-02$ & 0.68 & 0.750 \\
16 & Trehalose & 1.5 & $2.79 \mathrm{E}-02$ & 0.27 & 0.775 \\
17 & Naringenin & 1.5 & $3.55 \mathrm{E}-02$ & 0.45 & 0.785 \\
18 & Tyrosine & 1.5 & $3.55 \mathrm{E}-02$ & 1.58 & 0.770 \\
19 & Cyclohexane-1,2-diol & 1.6 & $3.99 \mathrm{E}-02$ & 47.19 & 0.655 \\
20 & Xylitol & 1.5 & $4.10 \mathrm{E}-02$ & 0.68 & 0.820 \\
21 & 24,25-Dihydrolanosterol & 1.5 & $4.13 \mathrm{E}-02$ & 0.59 & 0.780 \\
22 & Indolelactate & 1.5 & $4.21 \mathrm{E}-02$ & 0.54 & 0.730 \\
23 & Pentadecanoic acid & 1.4 & $4.47 \mathrm{E}-02$ & 2.48 & 0.640 \\
\hline
\end{tabular}

2 a. Variable Importance in Projection (VIP) values from Orthogonal Projections to Latent Structures Discriminant Analysis (PLS-

3 DA); b. Fold change of metabolites expression in model group compared to controls; c. ROC: receiver-operating characteristic 4 curve, AUC: area under the ROC curve. 
Table 2 (on next page)

Clustered metabolic pathways. 
1

\begin{tabular}{lccc}
\hline Metabolism pathway & Total & Hits & P-value \\
\hline Starch and sucrose metabolism & 18 & 2 & $1.75 \mathrm{E}-02$ \\
Pentose and glucuronate interconversions & 18 & 2 & $1.75 \mathrm{E}-02$ \\
Galactose metabolism & 27 & 2 & $3.77 \mathrm{E}-02$ \\
Phenylalanine, tyrosine and tryptophan biosynthesis & 4 & 1 & $4.57 \mathrm{E}-02$ \\
Steroid hormone biosynthesis & 85 & 3 & $7.15 \mathrm{E}-02$ \\
Steroid biosynthesis & 42 & 2 & $8.34 \mathrm{E}-02$ \\
Tyrosine metabolism & 42 & 2 & $8.34 \mathrm{E}-02$ \\
Valine, leucine and isoleucine biosynthesis & 8 & 1 & $8.94 \mathrm{E}-02$ \\
Ubiquinone and other terpenoid-quinone biosynthesis & 9 & 1 & $1.00 \mathrm{E}-01$ \\
Aminoacyl-tRNA biosynthesis & 48 & 2 & $1.05 \mathrm{E}-01$ \\
Phenylalanine metabolism & 10 & 1 & $1.11 \mathrm{E}-01$ \\
Glycerolipid metabolism & 16 & 1 & $1.71 \mathrm{E}-01$ \\
Citrate cycle (TCA cycle) & 20 & 1 & $2.09 \mathrm{E}-01$ \\
Glycolysis / Gluconeogenesis & 26 & 1 & $2.64 \mathrm{E}-01$ \\
Alanine, aspartate and glutamate metabolism & 28 & 1 & $2.81 \mathrm{E}-01$ \\
Glyoxylate and dicarboxylate metabolism & 32 & 1 & $3.14 \mathrm{E}-01$ \\
Amino sugar and nucleotide sugar metabolism & 37 & 1 & $3.54 \mathrm{E}-01$ \\
Valine, leucine and isoleucine degradation & 40 & 1 & $3.77 \mathrm{E}-01$ \\
Primary bile acid biosynthesis & 46 & 1 & $4.20 \mathrm{E}-01$ \\
Purine metabolism & 65 & 1 & $5.40 \mathrm{E}-01$ \\
\hline
\end{tabular}

2 Metabolomics pathway data analysis was performed by MetaboAnalyst. 
Table 3 (on next page)

Survival associated SLC transporters genes. 
1

\begin{tabular}{lcccc}
\hline Gene & P-value $^{\mathbf{a}}$ & HR $^{\mathbf{b}}$ & HR_low $^{\mathbf{c}}$ & HR_high $^{\mathbf{d}}$ \\
\hline SLC1A5 & $2.54 \mathrm{E}-02$ & 1.70 & 1.06 & 2.72 \\
SLC7A5 & $3.71 \mathrm{E}-04$ & 2.35 & 1.45 & 3.82 \\
SLC1A3 & $7.97 \mathrm{E}-04$ & 2.21 & 1.38 & 3.56 \\
SLC6A9 & $1.23 \mathrm{E}-02$ & 0.55 & 0.34 & 0.88 \\
SLC43A2 & $2.01 \mathrm{E}-03$ & 0.47 & 0.29 & 0.77 \\
SLC1A7 & $3.97 \mathrm{E}-03$ & 0.49 & 0.30 & 0.80 \\
SLC7A8 & $8.41 \mathrm{E}-03$ & 0.53 & 0.33 & 0.86 \\
SLC43A3 & $5.30 \mathrm{E}-04$ & 2.29 & 1.42 & 3.70 \\
SLC38A3 & $1.46 \mathrm{E}-02$ & 1.80 & 1.12 & 2.89 \\
\hline
\end{tabular}

2 a. Univariate survival analysis and multivariate survival analysis of SLC transporters genes, significance was

3 set at $\mathrm{P}<0.05$; b. HR, hazard ratio; c. lowest confidence interval; $d$. highest confidence interval. A confidence 4 interval of $95 \%$ was used. 